\title{
Vacancies for reversible insertion
}

Nat. Mater. http://doi.org/cfcf (2017)

Rechargeable batteries based on multivalent ions such as $\mathrm{Mg}^{2+}$ and $\mathrm{Al}^{3+}$ offer an attractive alternative to $\mathrm{Li}$-ion batteries, because these ions are more Earth-abundant and have the ability to transfer more electrons than $\mathrm{Li}^{+}$. However, the development of multivalent batteries has been largely hindered by the difficulty in intercalation or deintercalation of the multivalent ions into the electrodes, which is due to the strong electrostatic interactions between these ions and their hosts. Now, Peter Strasser, Damien Dambournet and colleagues design cation-deficient anatase $\mathrm{TiO}_{2}$, enabling reversible insertion of $\mathrm{Mg}^{2+}$ and $\mathrm{Al}^{3+}$.

The researchers fabricate anatase $\mathrm{TiO}_{2}$ electrodes with high concentrations of Ti vacancies (22\%) through the partial substitution of Ti cations by monovalent anions such as fluoride and hydroxide groups. The introduction of these anions charge-compensate for the creation of the Ti cations, making the resultant compounds stable. Using a comprehensive set of techniques such as high-energy X-ray and NMR, the researchers show that the number of $\mathrm{Mg}^{2+}$ ions that can be reversibly intercalated matches the concentration of the Ti vacancies, and the discharge capacity matches the number of inserted $\mathrm{Mg}^{2+}$ ions, indicating a two-electron redox reaction mechanism. In a three-electrode cell, which is useful for understanding how battery materials behave under operando conditions, the vacancy-rich anatase $\mathrm{TiO}_{2}$ electrodes display approximately six times higher discharge capacity $\left(155 \mathrm{mAh} \mathrm{g}^{-1}\right)$ than the stoichiometric anatase $\mathrm{TiO}_{2}$.

Changjun Zhang

Published online: 9 November 2017

https://doi.org/10.1038/s41560-017-0039-2 